\title{
Image-Guided High-Intensity Focused Ultrasound, A Novel Application for Interventional Nuclear Medicine?
}

\author{
Xinrui Zhang ${ }^{1}$, Lisa Landgraf ${ }^{1}$, Nikolaos Bailis ${ }^{2}$, Michael Unger ${ }^{1}$, Thies H. Jochimsen ${ }^{3}$, and Andreas Melzer ${ }^{1,4}$ \\ ${ }^{1}$ Innovation Center Computer Assisted Surgery (ICCAS), University of Leipzig, Leipzig, Germany; ${ }^{2}$ Department of Diagnostic and \\ Interventional Radiology, University of Leipzig, Leipzig, Germany; ${ }^{3}$ Department of Nuclear Medicine, Leipzig University Hospital, Leipzig, \\ Germany; and ${ }^{4}$ Institute of Medical Science and Technology (IMSaT), University of Dundee, Dundee, Scotland
}

\begin{abstract}
Image-guided high-intensity focused ultrasound (HIFU) has been increasingly used in medicine over the past few decades, and several systems for such have become commercially available. HIFU has passed regulatory approval around the world for the ablation of various solid tumors, the treatment of neurologic diseases, and the palliative management of bone metastases. The mechanical and thermal effects of focused ultrasound provide a possibility for histotripsy, supportive radiation therapy, and targeted drug delivery. The integration of imaging modalities into HIFU systems allows for precise temperature monitoring and accurate treatment planning, increasing the safety and efficiency of treatment. Preclinical and clinical results have demonstrated the potential of image-guided HIFU to reduce adverse effects and increase the quality of life postoperatively. Interventional nuclear image-guided HIFU is an attractive noninvasive option for the future.
\end{abstract}

Key Words: image-guided HIFU; targeted drug delivery; tumor ablation; hyperthermia; PET/MR image guidance

J Nucl Med 2021; 62:1181-1188

DOI: 10.2967/jnumed.120.256230

H igh-intensity focused ultrasound (HIFU) has gained clinical acceptance for therapeutic applications, especially as a thermal ablative treatment modality for benign uterine fibroids, bone metastases, and prostate carcinoma, as well as for essential and Parkinson tremors (1). HIFU ablation at temperatures above $55^{\circ} \mathrm{C}$ is considered noninvasive surgery without physical access to the target region, and clinical HIFU systems have received regulatory approval in Europe, America, and Asia (2). Additionally, HIFU-induced hyperthermia in a temperature range of $40^{\circ} \mathrm{C}-45^{\circ} \mathrm{C}$ for up to 60 min was reported as a sensitizer for ionizing radiation and a mediator of thermal-sensitive drug delivery in the treatment of solid tumors (3).

Compared with other clinically used techniques for thermal therapy (ablation) such as laser, radiofrequency, and microwaves, which generate heat through probes by electromagnetic waves, causing the rotation of water molecules and thus inducing heating, ultrasound is a mechanical-wave-based technique. Although it is possible to generate focused heat through microwaves or radiofrequencies, electromagnetic waves cannot be focused as precisely as ultrasound waves, and the penetration depth of electromagnetic waves is limited. HIFU

Received Nov. 13, 2020; revision accepted May 5, 2021.

For correspondence or reprints, contact Andreas Melzer (andreas.mezler@ uni-leipzig.de).

Published online June 4, 2021.

COPYRIGHT (C) 2021 by the Society of Nuclear Medicine and Molecular Imaging. provides adequate tissue penetration, ranging from 1 to $20 \mathrm{~cm}$, with specific focal spot. Therefore, it overcomes the limitation of electromagnetic wave-based techniques. Moreover, HIFU is a promising alternative and the only noninvasive technique for clinical ablation and hyperthermia (4).

In recent years, image-guided therapeutic interventions have become an increasingly important area in the oncology field, integrating radiologic imaging methods such as MRI, ultrasound imaging, CT, radiography, and PET with surgery and therapies (2). Evaluation of treatment with an adequate imaging protocol is essential as a clinical prognostic readout. The broad clinical applications of HIFU were reviewed by Izadifar et al. (5). In addition to that review, we provide clinical details of some of the most prevalent and important clinical HIFU applications, describing the relative systems for these clinical cases. An overview of the latest preclinical and clinical studies is given in this review.

\section{PRINCIPLES OF HIFU}

Regarding the application of HIFU, ultrasound waves are typically generated by piezoelectric material, and focusing is enabled either by a single focused transducer that bundles sound waves with the bowl-shape transducer (Fig. 1A) or by phased-array transducers via beam forming (Fig. 1B).

Numerous parameters should be taken into consideration from the physical aspect. For example, the selected frequency, in the range of $0.25-3 \mathrm{MHz}$ for medical applications, is related to penetration depth, indications, and the required acoustic intensity. For intraabdominal applications, a frequency of $1 \mathrm{MHz}$ is typically used to produce sufficiently high intensities for deep-seated tissues. Another important parameter is the acoustic impedance, which comprises sound speed in the medium and the density of the medium, affecting the transport of sound waves. When ultrasound waves propagate through tissues, sound waves are reflected at the interface if the acoustic impedances of 2 tissues are different. The reflection of sound waves can increase the signal display and help to distinguish the tissue interface (e.g., muscle and bone interface) with diagnostic ultrasound; however, reflection of sound waves is unexpected and may cause injuries in HIFU applications. Thus, air bubble-free coupling of the patient to the HIFU system is required to avoid reflection-induced skin damage. In this context, image guidance is of high importance to prevent scattering or the reflection of sound waves and avoid potential safety issues, especially for applications close to specific regions such as the lungs, intestines, and bones ( 6 ).

HIFU generates acoustic waves at a high energy level; absorption of acoustic energy creates a thermal effect in the tissues via mechanical friction. Cavitation, acoustic radiation force, acoustic streaming, 


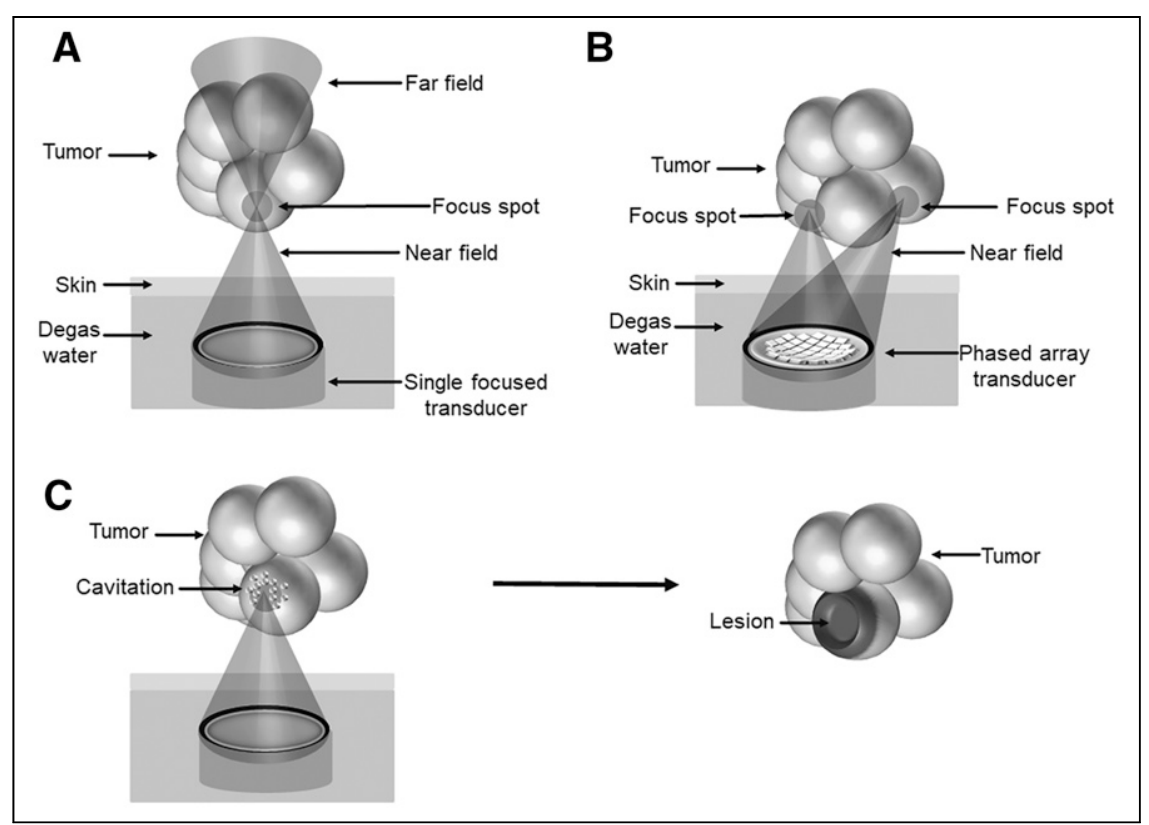

FIGURE 1. Schematic representation of HIFU ablation. (A) High level of sound energy in focal spot leads to heating of tissue to temperatures above $55^{\circ} \mathrm{C}$, resulting in coagulation with subsequent necrosis in tissue. Sensitive structures in near and far fields can be spared. (B) Ablation of large-volume tumors is realized by scanning focal zone through entire tumor using phased-array transducer via beam forming. (C) Histotripsy destroys tissue by induction of cavitation events, generating lesions in target region.
HIFU system into PET/MRI provides additional information on metabolism in the targeted tissue and allows precise HIFUtriggered drug delivery, especially for the treatment of neurodegenerative diseases.

Ultrasound imaging enables real-time anatomic imaging during HIFU treatment, offering an advantage in the treatment of moving organs. For example, tumor in the liver moves together with respiration during treatment and may lead to noise in MR images, but ultrasound imaging can overcome such limitations (9). The main drawback of ultrasound imaging-guided HIFU (USgHIFU) is the lack of real-time temperature mapping; the temperature and the thermal dose inside the target region cannot be determined. Since temperature feedback to control ultrasound power is not available, USgHIFU is usually conducted using a short sonication duration (e.g., 3-4 s) to avoid overheating and may result in incomplete ablation (10). Moreover, the high absorption and reflection of ultrasound by the skull bone are the main limitations of transcranial ultrasound and restrict the treatment of neurologic diseases using USgHIFU.

and shock waves are considered nonthermal mechanisms of HIFUinduced biologic effects. Cavitation describes the oscillation and collapse of gas-filled cavities in the acoustic field. The process is divided into stable (noninertial) and inertial cavitation (7). Enhanced membrane permeability via the induction of stable cavitation is named sonoporation, and this is applied to open the blood--brain barrier and support drug delivery into the central nervous system. In contrast, inertial cavitation is typically used for tissue destruction (histotripsy) or in combination with chemotherapy, which is the so-called sonodynamic therapy (8). Histotripsy describes the disintegration or liquefaction of tissue by short ultrasound shots, showing that HIFU is capable of generating pure mechanical damage to tissues (Fig. 1C).

\section{IMAGING GUIDANCE MODALITIES AND THERMOMETRY}

To ensure the noninvasiveness of HIFU application, imaging guidance plays essential roles in treatment planning, temperature monitoring, and follow-up after therapy. Currently, only singlemodality imaging guidance, either ultrasound imaging or MRI, is used in the clinic. Dual-modality imaging, such as PET/CT and PET/MRI, has drawn attention in recent years. Integration of the

\section{NOTEWORTHY}

- HIFU ablation has been used in the clinic for the noninvasive treatment of various diseases under ultrasound or MRI guidance.

- The combination of HIFU with other therapies showed promising advances in recent preclinical and clinical studies.

- Integration of the multifunctional imaging guidance modality into HIFU is required for precise treatment and accurate evaluation of treatment outcome.
In contrast, MRI-guided HIFU (MRgHIFU) provides spatial guidance in the targeted area for precise treatment planning. Nearly real-time temperature monitoring during treatment is feasible via MR thermometry using temperature-dependent proton resonance frequency shifting, T1 temperature mapping, or diffusion temperature mapping (11). However, MRgHIFU is more costly than USgHIFU, and the patient is required to be enclosed in a confined MRI scanner during treatment.

\section{CLINICAL APPLICATIONS OF IMAGE-GUIDED HIFU}

Image-guided HIFU systems have been developed and are used clinically for the treatment of various diseases. On the basis of physical parameters and available clinical systems with a focused spot of 1 $\mathrm{mm}-4 \mathrm{~cm}(5)$, HIFU treatment is typically conducted through several subsequential sonications (refers to the application of ultrasound energy) across the whole treated region. Here, we discuss some of the most commonly used clinical applications and relevant HIFU systems. Commercially available clinical HIFU systems are listed in Table 1.

\section{Ablation of Uterine Fibroids}

Uterine fibroids are the most common benign tumors in the uterus, showing great variability in shape, size, and localization. They occur in up to $80 \%$ of women during the reproductive period and are the leading indication for hysterectomy. Clinically relevant symptoms, including pelvic pain, dysmenorrhea, and dyspareunia, can be released with pharmacologic agents; however, adverse effects and a lack of adequate control are the main problems. Hospitalization and several weeks of recovery time are required after surgical resection (12).

The MRgHIFU system ExAblate 2000 (Insightec) was first approved by the U.S. Food and Drug Administration to treat uterine fibroids in 2004, and the Sonalleve system from Profound Medical was approved in Europe in 2011 (Fig. 2A). The Sonalleve system 
TABLE 1

Clinically Used HIFU Systems

\begin{tabular}{|c|c|c|c|}
\hline Device & Company & Location & Application field \\
\hline \multicolumn{4}{|l|}{ MRgHIFU } \\
\hline Low-intensity FUS pulsation & BrainSonix & Sherman Oaks, California & Neuromodulation \\
\hline Sonalleve & Profound Medical & Mississauga, Canada & Myomas, bone metastasis \\
\hline Tulsa-Pro & & & Prostate carcinoma \\
\hline SonoCloud & CarThera & Paris, France & Brain \\
\hline Targeted FUS & Image Guided Therapy & Pessac, France & Breast, uterus \\
\hline ExAblate 2000 & Insightec & Tirat Carmel, Israel & Uterus \\
\hline ExAblate 4000 & & & Essential tremor \\
\hline ExAblate 2100 CBS & & & Bones \\
\hline ExAblate Prostate & & & Prostate \\
\hline ArcBlate & EpiSonica & Hsichu City, Taiwan & Uterus, pancreas \\
\hline \multicolumn{4}{|l|}{ USgHIFU } \\
\hline Edison & HistoSonics & Ann Arbor, Michigan & Prostate \\
\hline HIFU Synthesizer & International Cardio Corp. & Minnetonka, Minnesota & Arteriosclerosis \\
\hline Mirabilis & Mirabilis Medical & Bothell, Washington & Uterus fibroids \\
\hline Sonablate & SonaCare Medical & Charlotte, North Carolina & Prostate \\
\hline Valvopulse & Cardiawave & Paris, France & Aortic valve stenosis \\
\hline EchoPulse & Theraclion & Malakoff, France & Fibroadema breast, thyroid \\
\hline Alpius 900 & Alpinion Medical Systems & Seoul, Korea & Uterus \\
\hline FEP-BY02 & $\begin{array}{l}\text { Beijing Yuande Bio-Medical } \\
\text { Engineering }\end{array}$ & Beijing, China & Pancreas \\
\hline JC200 & $\begin{array}{l}\text { Chongqing Haifu Medical } \\
\text { Technology Co. }\end{array}$ & Chongqing, China & Uterus, liver, bones, breast \\
\hline HIFUNIT 9000 & $\begin{array}{l}\text { Shanghai A\&S Science Technology } \\
\text { Development Co. }\end{array}$ & Shanghai, China & Pancreas \\
\hline PRO2008 & Shenzhen PRO HITU Medical Co. & Shenzhen, China & Uterus \\
\hline Ablatherm & EDAP-TMS & Lyon, France & Prostate \\
\hline Focal One & & & \\
\hline
\end{tabular}

is introduced here as an example. The phased-array transducer consists of 256 elements that can be controlled independently, allowing beam forming. Formation of the ultrasound beam is established through electronic activation of each element separately at different phases, thereby creating a target zone with variable sizes and orientations. Treatment planning is based on the T2-weighted MR image, and the treatment volume within the target fibroids is determined in 3 orthogonal planes. Each sonication increases the temperature to $60^{\circ} \mathrm{C}-85^{\circ} \mathrm{C}$ in a matter of seconds and causes coagulative necrosis. The treatment outcome is evaluated with the nonperfused volume via contrast-medium-supported T1-weighted images (Fig. 2B). Nonperfused volume describes the tissue volume without blood flow and correlates with a fibroid volume reduction. In most cases, only a few hours of clinical monitoring are necessary, and the patients can continue with their daily activities after HIFU treatment.

Compared with traditional surgery, HIFU treatment is less invasive, avoids general anesthesia, and has the advantages of low morbidity, fewer complications, and a shorter recovery time. The symptoms subside either entirely or almost entirely 3-12 mo after successful therapy (nonperfused volume $>60 \%$ ). Various studies have shown a reduction in fibroid-associated complaints and an improved quality of life. The most significant advantages of image-guided HIFU over myomectomy are the higher pregnancy rate and the significantly reduced interval between pregnancy and treatment. The incidence of uterine rupture is rare in pregnant patients who have received HIFU ablation previously but should be considered to avoid serious adverse effects (13). The disadvantages are limited to the relatively long treatment duration of $2-4 \mathrm{~h}$ and the latency period until freedom from symptoms. Notably, the thick abdominal subcutaneous fat and the abdominal scar from previous surgery are associated with thermal injury and influence the risk of unsuccessful HIFU ablation of uterine fibroids (14). This complication can be avoided by careful preparation of the patients.

\section{Ablation Treatment of Essential Tremors}

Essential tremor is a neurologic disorder that causes rhythmic shaking, most often in the hands, and has a great impact on the quality of life. Some patients may not be able to work because of uncontrolled movements (15). Medications can be used at the early stage but are insufficient when the tremor progresses. Surgery is highly effective; however, open neurosurgical operation is associated with risks such as serious neurologic deficits and infections.

The ExAblate Neuro system from Insightec (Fig. 3A), compatible with both GE Healthcare and Siemens MRI scanners, has been 


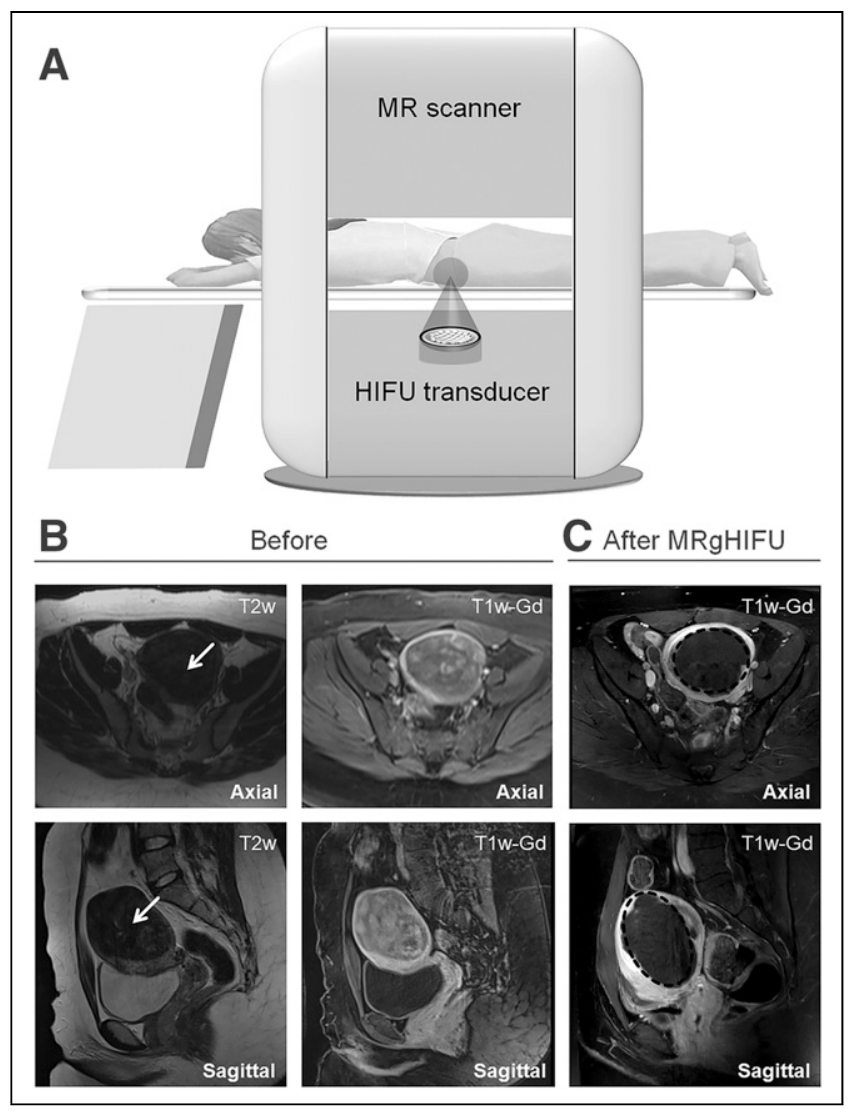

FIGURE 2. MRgHIFU for uterine fibroid treatment. (A) Schematic drawing of MRgHIFU treatment of uterine fibroids with Sonalleve or ExAblate 2000. Diagnostic T2- and T1-weighted contrast-enhanced (gadolinium) images are shown of patients with hyper- and dysmenorrhea and Funaki type 1 fibroid before (B, white arrow) and after ( $\mathrm{C}$, black circle) MRgHIFU. Fibroid volume is $184 \mathrm{~mL}$; treatment duration is up to $4 \mathrm{~h} . \mathrm{w}=$ weighted.

approved for the treatment of essential and Parkinson tremors and pain treatment. The helmet-shaped phased-array transducer contains 1,024 individual elements, enabling the focus of ultrasound beams in deep brain targets without a surgical incision. A sealed degassed water system is used for coupling and cooling during treatment. Clinical systems for the blood-brain barrier opening operate at $250 \mathrm{kHz}$, and systems for ablation while penetrating the skull operate at $720 \mathrm{kHz}$. The frequencies used for neurologic applications are lower than those used for body applications.

A pivotal clinical trial, conducted at 8 international centers for the treatment of essential tremors using the ExAblate transcranial MRgHIFU system (NCT01827904), is in the final phase of a 5-y follow-up. Improvement in the hand-tremor score was observed 3 mo after treatment (16), and continued beneficial effects in tremor control and the quality of life, with no progressive complications, were reported during follow-up for up to $4 \mathrm{y}$ (17).

\section{Ablation of Thyroid Nodules and Breast Fibroadenomas}

Thyroid nodules are common and are mostly benign, although some become large and symptomatic (18). Surgical resection is the standard treatment but may cause bleeding, hematoma, and infection, and general anesthesia is required. HIFU allows nonsurgical ablation and offers an alternative treatment for benign thyroid nodules (19).

To date, the EchoPulse (Theraclion; Fig. 3B) is the only commercially available device for the treatment of thyroid nodules. It consists of an ultrasound imaging system and a HIFU delivery system. The linear-array imaging probe uses frequencies of 7.5-12 $\mathrm{MHz}$ as guidance, and the treatment is performed with the HIFU probe at $3 \mathrm{MHz}$ with an acoustic power of up to $125 \mathrm{~W}$.

The first-in-humans trial of HIFU ablation of thyroid nodules was performed in France (20). The pathologic analysis showed significant nodule destruction, and histologic examination demonstrated that ablated lesions were confined to the target region, without damage to surrounding tissues. The safety and efficiency of the treatment were confirmed by Prakash et al. (21). The diameters of the thyroid nodules were reduced at 6 mo after treatment without complications (e.g., skin burns and hematoma), demonstrating the great potential of HIFU in the treatment of thyroid nodules.

In addition, breast fibroadenoma is another indication that can be treated using the EchoPluse system. A clinical trial was conducted in which patients were treated at an intensity of $40 \mathrm{~W}$ for 38 min under local anesthesia; a success rate of $89 \%$ was reported (22).

\section{Ablation of Prostate Cancer}

Prostate cancer is the second-most-common cancer in men worldwide (23). Conventional clinical therapies such as hormone therapy (chemical castration), radiation therapy (RT), and prostatectomy have side effects, including loss of sexual function, injury of the neurovascular bundle, and induction of urinary incontinence. Hence, local treatment options are gaining increasing interest. Laser ablation, cryoablation, and irreversible electroporation are used for prostate ablation (24), all of these applications require the insertion of probes, with associated side effects.

As the first approved MRgHIFU system for prostate cancer ablation, the TUSLA-Pro system (Fig. 3C) developed by Profound Medical combines real-time MRI and robotically driven transurethral ultrasound with temperature control. The individually controlled elements in the transurethral applicator generates directional thermal ultrasound, and an endorectal water-cooling device protects the rectum during treatment. The treated prostate tumor volume is limited to $40 \mathrm{~cm}^{3}$ for most transrectal HIFU systems; a total ablation of larger volumes is difficult because of the limited penetration depth. In contrast, the transurethral applicator has advantages for ablation of prostate lesions up to $200 \mathrm{~cm}^{3}$ by continuous rotation. The TUSLA-Pro system can be integrated into MRI scanners from various vendors; clinical feasibility and safety have been proven (25). The first integration with the PET/MRI scanner at OncoRay, University of Dresden, Germany, was realized within our SonoRay BMBF Project (https://www.oncoray.de/research/sono-ray), and a pilot clinical study is being conducted.

A multicenter phase I clinical trial of MRgHIFU whole-gland prostate ablation (NCT01686958) was conducted using the TUSLA-Pro system, and the radiologic findings were reported by Bonekamp et al. (26). Chin et al. (27) reported the oncologic outcomes and quality of life and stated that the erectile function of the patients was recovered after $12 \mathrm{mo}$. Klotz et al. (28) reported similar outcomes from a prospective trial (NCT02766543) with 115 patients in 2021, whereby the prostate-specific antigen decreased in $96 \%$ of the patients, and erections were maintained in $75 \%$ of the patients after 12 mo. The clinical data suggest that TUSLAPro is an effective tool for prostate cancer ablation, with minimal impact on the quality of life.

The novel HIFU hemiablation procedure targets only the cancerous tissue of the prostate, enabling the healthy tissue to be spared, which shows the potential to increase the effectiveness and safety of treatment compared with resection of the entire gland. Ganzer et al. (29) reported a multicenter phase II trial using the HIFU 


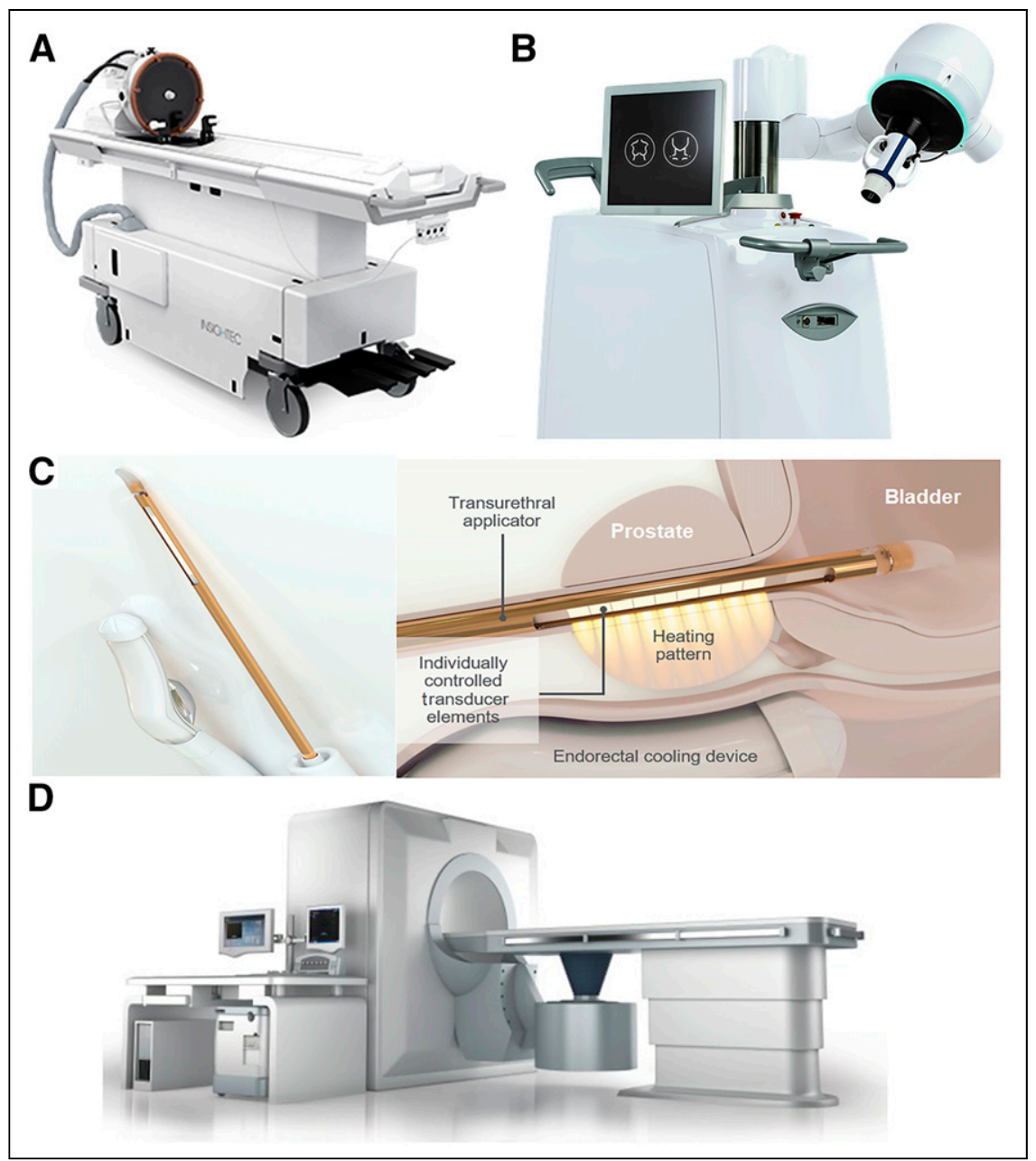

FIGURE 3. Clinically approved HIFU systems. (A) MRgHIFU system for neurologic application (EXAblate Neuro). Patient table includes FUS transducer mounted on mechanical positioning unit with MRI-compatible head frame that can be docked to MRI scanner. (B) EchoPulse USgHIFU system for treatment of thyroid nodules and breast fibroadenomas. (C) Transurethral TUSLA-Pro MRgHIFU ablation system for prostate cancer. (D) JC USgHIFU system for solid tumor ablation of all types of soft tissue. (Images courtesy of Insightec, Theraclion, Profound Medical, and Chongqing Haifu.) started in 2020 to investigate the pain palliation effect of MRgHIFU as an alternative or addition to RT (NCT04307914).

For successful HIFU treatment, patient selection is critical. Lesions of the spinal column should be carefully assessed because of the adjacent critical nerve structures. Notably, blood perfusion in highly vascularized lesions will lead to a heat-sink effect, and embolization should be considered in these cases.

\section{Ablation of Pancreatic Cancer}

Pancreatic cancer is aggressive, with a poor 5-y survival rate of $8 \%$. Symptoms include jaundice, malaise, weight loss, and loss of appetite, reducing patients' quality of life severely. Cancer-related abdominal or back pain is experienced in most patients, and established chemotherapy or radiochemotherapy has a limited antitumor effect and pain control duration (33).

The most frequently used clinical USgHIFU system, JC, was developed by Haifu (Chongqing; Fig. 3D) and has been approved for the treatment of uterine fibroids; pancreatic cancer; and liver, bone, and breast tumors. The system has a 0.8 $\mathrm{MHz}$ single transducer integrated into the patient table, and fronted acoustic lenses allow focus-length adjustment. An ultrasound-imaging device $(1-8 \mathrm{MHz})$ is equipped for real-time guidance.

A clinical trial in 2018 showed a reduction in tumor volume of $58 \%$ after $6 \mathrm{mo}$ and a longer median overall survival (16.2 mo) than for patients who received chemotherapy or radiochemotherapy (34). An improvement in quality of life was reported in 2021, with physical, emotional, and social functioning hemiablation approach. The 1-y follow-up indicated a significant reduction in prostate-specific antigen from 6.2 to $2.9 \mathrm{ng} / \mathrm{mL}$. Patients had a preserved quality of life after treatment. The lack of long-term follow-up on oncologic control and functional outcomes is the main obstacle to encouraging patients to choose this approach.

\section{Relief of Pain from Bone Metastases}

Bone is a common site of solid tumor metastases. Symptoms, including localized pain, pathologic fractures, and functional deficits, may occur early in the metastatic course. The treatment of painful metastases requires an interdisciplinary approach. There are many procedures available for treatment. The most common is RT at a single fraction of $8 \mathrm{~Gy}$ or at 10 fractions of $3 \mathrm{~Gy}$ each; however, a high toxicity rate was reported (30).

In a phase III study (31), pain relief was observed in two thirds of the respondents within $3 \mathrm{~d}$ after MRgHIFU treatment. Similarly, $89 \%$ of the patients experienced pain reduction, $72 \%$ of whom were completely pain-free, as reported by Napoli et al. (32). These results highlight the capability of HIFU in pain management of bone metastases, with rapid, successful, and durable effects. A new multicenter, 3 -armed, randomized controlled trial including more than 200 patients increasing while the rate of side effects remained low, demonstrating that HIFU is a worthwhile supportive treatment for advanced pancreatic cancer (35).

\section{INNOVATIVE APPLICATIONS OF IMAGE-GUIDED FOCUSED ULTRASOUND (FUS)}

In addition to the image-guided HIFU ablation described above, FUS at various intensities offers advantages in many other potential applications. The following section presents selected preclinical and clinical studies of FUS as a single or adjuvant therapy.

\section{Histotripsy}

As mentioned previously, dense energetic bubble clouds can be produced at the focus point by very short, infrequent, highamplitude bursts of acoustic waves that mechanically fractionate the tissue into subcellular components in histotripsy (36). Compared with thermal applications, histotripsy avoids overheating and the heat-sink effect in the targeted tissues, and tissue fragments are more easily absorbed after treatment.

Several recent studies showed the feasibility of using histotripsy to create precise lesions at the desired location, such as the liver, 
prostate, or brain (36). Notably, lesions can be created through the ribs safely throughout the entire liver (37). The first-in-humans study using cavitation histotripsy for the treatment of prostate enlargement was reported in 2018. An improvement in lower-urinary-tract symptoms was observed, with no intraoperative side effects. However, the treatment outcome was not as significant as expected, because of the technical limitations of the FUS device (38). The development of a precisely controlled histotripsy FUS system is necessary.

Histotripsy is a neurosurgical option (39) in which lesions with a dimension of up to $1 \mathrm{~cm}$ are created in the porcine cortex without thermally induced damage to the surrounding neurons. Moreover, histotripsy carries the potential to overcome the limitations of current thrombolytic drugs and to shorten the treatment time. In vitro and in vivo experiments have shown that FUS histotripsy thrombolysis can cause a direct mechanical breakdown of clots and achieve blood flow restoration noninvasively (40).

\section{FUS-Mediated Drug Delivery}

The systemic toxicity of chemotherapy is the main challenge in clinical oncology and may induce adverse effects such as hair loss, anemia, nausea, and vomiting. The encapsulation of chemotherapeutic drugs in carriers or the triggering of drug delivery in the target tissue with external physical stimulation has been considered to improve treatment efficiency and reduce adverse effects. FUSmediated targeted drug delivery based on mechanical and thermal mechanisms has become a topic of intensive research in the last $20 \mathrm{y}$. The drug carriers can be opened in situ and allow controlled drug release in specific regions with FUS exposure, and the permeability of the cell membrane and the drug uptake can be enhanced as well (41). A preclinical study reporting on the combination of the chemotherapeutic agent gemcitabine with FUS showed that the treatment led to a reduction in tumor growth compared with a single treatment in the xenograft pancreatic cancer model (42).

In addition to chemotherapeutic drugs, ultrasound-mediated gene delivery provides a new strategy for cancer treatment. The typical ultrasound parameters used for gene delivery are frequencies of 1-2 MHz, intensities of $0.1-3 \mathrm{~W} / \mathrm{cm}^{2}$, and short durations of 2-8 $\mathrm{s}$, with ultrasound contrast agent microbubbles usually being added to enhance the sonoporation effect (43). Noroozian et al. (44) demonstrated the FUS-mediated recombinant adeno-associated viral vector across the blood-brain barrier into the brain parenchyma, which resulted in efficient gene transduction and expression in both mouse and rat models.

A single-center phase 1 trial (NCT02181075) was conducted in the United Kingdom to analyze the safety and feasibility of FUS hyperthermia-triggered doxorubicin delivery from thermosensitive liposomes (45). Thermosensitive liposomal doxorubicin was administrated by intravenous infusion, followed by FUS exposure at a single target liver tumor. The concentration of doxorubicin inside the target tumor was increased by 3.7-fold immediately after FUS treatment, compared with the group without FUS.

\section{FUS Supporting RT}

$\mathrm{RT}$ is one of the most commonly used treatment modalities in the clinic. Various biologic mechanisms are involved in enhancing the antitumor effects of RT, including inhibition of DNA damage repair, induction of apoptosis, and changes in the tumor microenvironment (46). A few clinical studies have shown that FUS is a sensitizer of RT in different cancers, including those of the head and neck, breast, prostate, and central nervous system (3).
A phase I study by Chu et al. (47) reported combined treatment with MRgHIFU hyperthermia, RT, and chemotherapy for recurrent rectal cancer. Patients received daily fractioned RT (total dose, 30.6 Gy) and oral capecitabine. HIFU hyperthermia was performed at a mean temperature of $42.5^{\circ} \mathrm{C}$ for 30 min immediately before RT on days 1,8 , and 15. No intraoperative complications, serious adverse effects, or unexpected tissue damage attributed to HIFU hyperthermia were observed, showing the safety and feasibility of the treatment. Another phase II study reported a $7-y$ overall survival rate of $94 \%$ in advanced prostate cancer patients after combined treatment with transrectal ultrasound hyperthermia, RT, and androgen suppression (48).

A better understanding of ultrasound radiosensitization mechanisms is necessary, although benefits have been shown in several clinical studies. The significant radioadditive effect of FUS in prostate cancer was demonstrated in our in vitro study $(49,50)$. It is critical to optimize the combination-treatment protocols to increase treatment efficiency in terms of treatment sequences, intervals, thermal doses, and radiation doses. The integration of FUS into RT and the administration of both treatments simultaneously may improve the radiosensitization effect further.

\section{FUS-Mediated Immunotherapy}

Immunotherapy has changed cancer treatment strategies, aiming to improve the antitumor immune response and directly kill cancer cells (51). The immune system is activated or the activation process is boosted by immunotherapeutic agents such as checkpoint inhibitors to attack cancer cells through natural mechanisms and avoid relative side effects.

FUS provides the possibility of delivering immunotherapy agents, and FUS administration, especially the mechanisms of cavitation, is considered a stimulator for the immune system (52). Eranki et al. (53) reported that the mechanical fractionation of neuroblastoma tumors using HIFU significantly increases cellular immunity, and the combination of HIFU and checkpoint inhibitors induces systemic immunity and dramatically enhances survival. More experimental and clinical evidence of FUS-induced immune effects was systematically reviewed by van den Bijgaart et al. (54). However, the safety of FUS-induced immune therapy is the main obstacle for clinical translation because of tumor heterogeneity. The underlying mechanisms of the interactions between ultrasound and cells are of vital importance, and a better understanding is needed before clinical trials.

\section{Neuromodulation by Low-Intensity FUS}

Transcranial FUS application in neurologic diseases is of particular interest. The potential indications include Alzheimer disease, depression, obsessive-compulsive disorder, epilepsy, and Huntington disease. FUS-mediated neuromodulation is performed at low intensities and for short durations (55). Mechanical effects are considered the main driver for neuron stimulation, since the temperature increase is limited at the target.

Storz Medical has introduced the CE (Conformité Européenne)marked system Neurolith for single-pulse-wave treatment of Alzheimer disease, with promising initial clinical results (56). Low-intensity FUS with short bursts is a promising method for the safe stimulation or inhibition of neurons in future clinical neurologic applications and will not require that the patient's hair be shaved. A new clinical FUS system operating at diagnostic sound pressure for neuromodulation has been developed collaboratively by the Innovation Center Computer Assisted Surgery and the Fraunhofer IBMT and is currently being validated at the Max Planck Institute for 


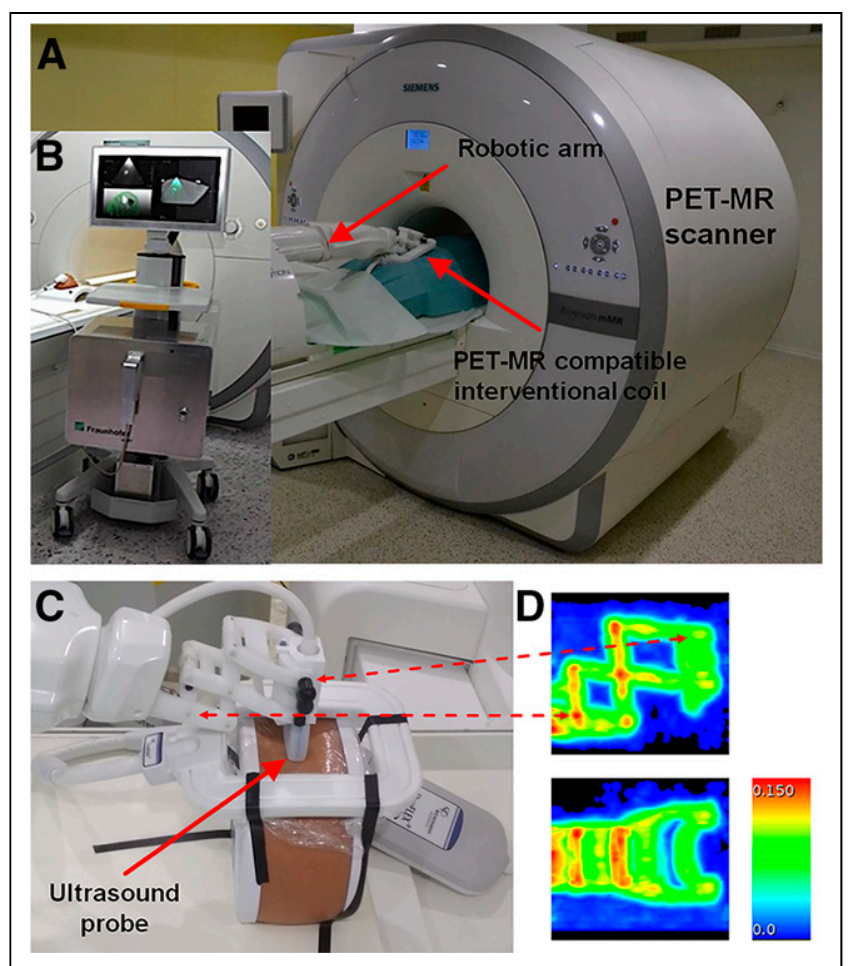

FIGURE 4. Integration of Innomotion robotic arm (InnoMedic $\mathrm{GmbH}$ ) into Biograph mMR PET/MRI scanner (Siemens Healthineers). (A) Robotic arm was modified to fit into PET/MRI system holding ultrasound imaging probe. (B) MRI-compatible FUS system Diphas (Fraunhofer IBMT) can be integrated into PET/MRI scanner. (C) Three-dimensionally printed adaptor and fixation parts were manufactured to hold ultrasound transducer. (D) Attenuation map of robotic arm visualized by 2 perpendicular maximumintensity projections of PET annihilation photons (in 1/cm) with linear attenuation coefficient of $511 \mathrm{keV}$, obtained by stand-alone PET scanner equipped with ${ }^{68} \mathrm{Ge}-/ 68 \mathrm{Ga}$ rod sources.

Cognitive and Neurosciences in Leipzig. The preliminary results were reported at the first Focused Ultrasound Neuromodulation Symposium in Oxford, England, on September 23-25, 2019. Further work is necessary to explore the ultrasound parameters and to develop targeted neuromodulation methods for different brain regions.

\section{NOVEL TECHNIQUES FOR POTENTIAL IMAGE-GUIDED HIFU APPLICATIONS}

Various studies have been conducted to integrate multifunctional imaging modalities into the FUS system to improve the safety, treatment efficiency, and accuracy of the outcome assessment. PET/MRI was also reported to detect the local recurrence of prostate cancer patients after HIFU ablation, which is not detectable by MRI. Moreover, PET/MR images could be used to optimize targeting of a secondary HIFU ablation and could improve patient survival (57). Novel tracers, such as those utilizing the prostate-specific membrane antigen, have shown promising results in patients for the detection of prostatic cancer using PET/MRI (58). In combination with HIFU, such tracers may allow for a 1-stop-shop focal treatment.

A robotic assistance system was previously integrated into an MRI scanner to provide precise and reproducible instrument positioning (59). To realize precise FUS positioning under PET/MRI guidance, we are working toward integrating a robotic arm system with an MRI-compatible FUS transducer into the clinical PET/ MRI scanner (Fig. 4) (60). This new concept expands the application field of image-guided FUS interventions, may provide clinical feasibility, and will soon allow precise treatment.

\section{CONCLUSION}

Imaged-guided HIFU is a promising noninvasive, nonaccess clinical method that has been proven to be efficient and safe in several studies. It has been approved in all major markets around the world for the treatment of uterine fibroids, bone metastases, liver tumors, brain cancer, and prostate cancer. The advantages of the method are its noninvasiveness, absence of ionizing radiation, comparatively low complication rate, and low side effect rate, with a correspondingly low impairment of quality of life. With a transcranial HIFU system, the treatment of essential tremors and Parkinson-induced tremors is clinically approved, and studies that are currently under way have had promising initial results in neurologic diseases. The potential of HIFU has been demonstrated for various medical applications to improve other treatment modalities such as chemotherapy and ER and to support drug delivery. Imaging innovation together with HIFU treatment may provide a new strategy for future clinical applications.

\section{DISCLOSURE}

The work described was supported by research grants funded by the German Ministry of Science and Technology BMBF project SONO-RAY (03Z1L511). No other potential conflict of interest relevant to this article was reported.

\section{ACKNOWLEDGMENTS}

We thank the Departments of Nuclear Medicine and Radiation Therapy of the Leipzig University Hospital for supporting some of the work mentioned (further results will be published elsewhere), and we thank the Saxon Incubator for Clinical Translation and the Fraunhofer Institute for Cell Therapy and Immunology for supporting the preclinical experiments.

\section{REFERENCES}

1. Kennedy JE. High-intensity focused ultrasound in the treatment of solid tumours. Nat Rev Cancer. 2005;5:321-327.

2. Siedek F, Yeo SY, Heijman E, et al. Magnetic resonance-guided high-intensity focused ultrasound (MR-HIFU): technical background and overview of current clinical applications (part 1). Rofo. 2019;191:522-530.

3. Zhu L, Altman MB, Laszlo A, et al. Ultrasound hyperthermia technology for radiosensitization. Ultrasound Med Biol. 2019;45:1025-1043.

4. Jolesz FA. MRI-guided focused ultrasound surgery. Annu Rev Med. 2009;60: $417-430$.

5. Izadifar Z, Izadifar Z, Chapman D, Babyn P. An introduction to high intensity focused ultrasound: systematic review on principles, devices, and clinical applications. JClin Med. 2020;9:460

6. McWilliams JP, Lee EW, Yamamoto S, Loh CT, Kee ST. Image-guided tumor ablation: emerging technologies and future directions. Semin Intervent Radiol. 2010;27: 302-313.

7. Prentice P, Cuschierp A, Dholakia K, Prausnitz M, Campbell P. Membrane disruption by optically controlled microbubble cavitation. Nat Phys. 2005;1:107-110.

8. Rosenthal I, Sostaric JZ, Riesz P. Sonodynamic therapy: a review of the synergistic effects of drugs and ultrasound. Ultrason Sonochem. 2004;11:349-363.

9. Orsi F, Arnone P, Chen WZ, Zhang LA. High intensity focused ultrasound ablation: a new therapeutic option for solid tumors. J Cancer Res Ther. 2010;6:414 420.

10. Orsi F, Monfardini L, Bonomo G, Krokidis M, Della Vigna P, Disalvatore D. Ultrasound guided high intensity focused ultrasound (USgHIFU) ablation for uterine fibroids: do we need the microbubbles? Int J Hyperthermia. 2015;31:233-239.

11. Samset E. Temperature mapping of thermal ablation using MRI. Minim Invasive Ther Allied Technol. 2006;15:36-41.

12. Stewart EA. Clinical practice. uterine fibroids. N Engl J Med. 2015;372:1646-1655. 
13. Wu G, Li R, He M, et al. A comparison of the pregnancy outcomes between ultrasound-guided high-intensity focused ultrasound ablation and laparoscopic myomectomy for uterine fibroids: a comparative study. Int J Hyperthermia. 2020;37: 617-623.

14. Duc NM, Keserci B. Review of influential clinical factors in reducing the risk of unsuccessful MRI-guided HIFU treatment outcome of uterine fibroids. Diagn Interv Radiol. 2018;24:283-291.

15. Hopfner F, Deuschl G. Managing essential tremor. Neurotherapeutics. 2020;17: 1603-1621.

16. Elias WJ, Lipsman N, Ondo WG, et al. A randomized trial of focused ultrasound thalamotomy for essential tremor. N Engl J Med. 2016;375:730-739.

17. Park YS, Jung NY, Na YC, Chang JW. Four-year follow-up results of magnetic resonance-guided focused ultrasound thalamotomy for essential tremor. Mov Disord. 2019;34:727-734

18. Durante C, Costante G, Lucisano G, et al. The natural history of benign thyroid nodules. JAMA. 2015;313:926-935.

19. Lang BHH, Wu ALH. High intensity focused ultrasound (HIFU) ablation of benign thyroid nodules: a systematic review. J Ther Ultrasound. 2017;5:11.

20. Esnault O, Franc B, Menegaux F, et al. High-intensity focused ultrasound ablation of thyroid nodules: first human feasibility study. Thyroid. 2011;21:965-973.

21. Prakash PS, Oh HB, Tan WB, Parameswaran R, Ngiam KY. The efficacy and safety of high-intensity focused ultrasound (HIFU) therapy for benign thyroid nodules: a single center experience from Singapore. World J Surg. 2019;43:1957-1963.

22. Hahn M, Fugunt R, Schoenfisch B, et al. High intensity focused ultrasound (HIFU) for the treatment of symptomatic breast fibroadenoma. Int $J$ Hyperthermia. 2018;35: $463-470$.

23. Barsouk A, Padala SA, Vakiti A, et al. Epidemiology, staging and management of prostate cancer. Med Sci (Basel). 2020;8:28.

24. Valerio M, Cerantola Y, Eggener SE, et al. New and established technology in focal ablation of the prostate: a systematic review. Eur Urol. 2017;71:17-34.

25. Eggener S, Koch M, Penson D, et al. Pivotal trial of MRI-guided transurethral ultrasound ablation in men with localized prostate cancer: two-year follow-up [abstract]. $J$ Urol. 2020;203(suppl):e369.

26. Bonekamp D, Wolf MB, Roethke MC, et al. Twelve-month prostate volume reduction after MRI-guided transurethral ultrasound ablation of the prostate. Eur Radiol. 2019;29:299-308.

27. Chin JL, Billia M, Relle J, et al. Magnetic resonance imaging-guided transurethral ultrasound ablation of prostate tissue in patients with localized prostate cancer: a prospective phase 1 clinical trial. Eur Urol. 2016;70:447-455.

28. Klotz L, Pavlovich CP, Chin J, et al. Magnetic resonance imaging-guided transurethral ultrasound ablation of prostate cancer. J Urol. 2021;205:769-779.

29. Ganzer R, Hadaschik B, Pahernik S, et al. Prospective multicenter phase II study on focal therapy (hemiablation) of the prostate with high intensity focused ultrasound. $J$ Urol. 2018;199:983-989.

30. Loi M, Nuyttens JJ, Desideri I, Greto D, Livi L. Single-fraction radiotherapy (SFRT) for bone metastases: patient selection and perspectives. Cancer Manag Res. 2019;11: 9397-9408.

31. Hurwitz MD, Ghanouni P, Kanaev SV, et al. Magnetic resonance-guided focused ultrasound for patients with painful bone metastases: phase III trial results. J Natl Cancer Inst. 2014;106:dju082.

32. Napoli A, Anzidei M, Marincola BC, et al. Primary pain palliation and local tumor control in bone metastases treated with magnetic resonance-guided focused ultrasound. Invest Radiol. 2013;48:351-358.

33. Carrato A, Falcone A, Ducreux M, et al. A systematic review of the burden of pancreatic cancer in Europe: real-world impact on survival, quality of life and costs. J Gastrointest Cancer. 2015;46:201-211.

34. Marinova M, Huxold HC, Henseler J, et al. Clinical effectiveness and potential survival benefit of US-guided high-intensity focused ultrasound therapy in patients with advanced-stage pancreatic cancer. Ultraschall Med. 2019;40:625-637.

35. Marinova M, Feradova H, Gonzalez-Carmona MA, et al. Improving quality of life in pancreatic cancer patients following high-intensity focused ultrasound (HIFU) in two European centers. Eur Radiol. January 23, 2021 [Epub ahead of print].

36. Khokhlova VA, Fowlkes JB, Roberts WW, et al. Histotripsy methods in mechanical disintegration of tissue: towards clinical applications. Int J Hyperthermia. 2015;31: $145-162$.
37. Vlaisavljevich E, Owens G, Lundt J, et al. Non-invasive liver ablation using histotripsy: preclinical safety study in an in vivo porcine model. Ultrasound Med Biol. 2017;43:1237-1251.

38. Schuster TG, Wei JT, Hendlin K, Jahnke R, Roberts WW. Histotripsy treatment of benign prostatic enlargement using the Vortx $\mathrm{R}_{\mathrm{x}}$ system: initial human safety and efficacy outcomes. Urology. 2018;114:184-187.

39. Sukovich JR, Cain CA, Pandey AS, et al. In vivo histotripsy brain treatment. JNeurosurg. October 1, 2018 [Epub ahead of print].

40. Shi A, Lundt J, Deng Z, et al. Integrated histotripsy and bubble coalescence transducer for thrombolysis. Ultrasound Med Biol. 2018;44:2697-2709.

41. Jain A, Tiwari A, Verma A, Jain SK. Ultrasound-based triggered drug delivery to tumors. Drug Deliv Transl Res. 2018;8:150-164.

42. Park EJ, Ahn YD, Lee JY. In vivo study of enhanced chemotherapy combined with ultrasound image-guided focused ultrasound (USgFUS) treatment for pancreatic cancer in a xenograft mouse model. Eur Radiol. 2018;28:3710-3718.

43. Yoon CS, Park JH. Ultrasound-mediated gene delivery. Expert Opin Drug Deliv. 2010;7:321-330

44. Noroozian Z, Xhima K, Huang Y, et al. MRI-guided focused ultrasound for targeted delivery of rAAV to the brain. Methods Mol Biol. 2019;1950:177-197.

45. Lyon PC, Gray MD, Mannaris C, et al. Safety and feasibility of ultrasound-triggered targeted drug delivery of doxorubicin from thermosensitive liposomes in liver tumours (TARDOX): a single-centre, open-label, phase 1 trial. Lancet Oncol. 2018;19:1027-1039.

46. Rao W, Deng ZS, Liu J. A review of hyperthermia combined with radiotherapy/chemotherapy on malignant tumors. Crit Rev Biomed Eng. 2010;38:101-116.

47. Chu W, Huang Y, Pichardo S, et al. A phase I study of MR-hifu hyperthermia (HT) with radiation (RT) and chemotherapy (CT) for recurrent rectal cancer [abstract]. $J$ Glob Oncol. 2019;5:78.

48. Hurwitz MD, Hansen JL, Prokopios-Davos S, et al. Hyperthermia combined with radiation for the treatment of locally advanced prostate cancer: long-term results from Dana-Farber Cancer Institute study 94-153. Cancer. 2011;117:510-516.

49. Hu S, Zhang X, Unger M, Patties I, Melzer A, Landgraf L. Focused ultrasoundinduced cavitation sensitizes cancer cells to radiation therapy and hyperthermia. Cells. 2020;9:2595.

50. Zhang X, Bobeica M, Unger M, et al. Focused ultrasound radiosensitizes human cancer cells by enhancement of DNA damage. Strahlenther Onkol. April 22, 2021 [Epub ahead of print].

51. Riley RS, June CH, Langer R, Mitchell MJ. Delivery technologies for cancer immunotherapy. Nat Rev Drug Discov. 2019;18:175-196.

52. Mauri G, Nicosia L, Xu Z, et al. Focused ultrasound: tumour ablation and its potential to enhance immunological therapy to cancer. Br J Radiol. 2018;91:20170641.

53. Eranki A, Srinivasan P, Ries M, et al. High-intensity focused ultrasound (HIFU) triggers immune sensitization of refractory murine neuroblastoma to checkpoint inhibitor therapy. Clin Cancer Res. 2020;26:1152-1161.

54. van den Bijgaart RJ, Eikelenboom DC, Hoogenboom M, Futterer JJ, den Brok MH, Adema GJ. Thermal and mechanical high-intensity focused ultrasound: perspectives on tumor ablation, immune effects and combination strategies. Cancer Immunol Immunother. 2017;66:247-258.

55. Naor O, Krupa S, Shoham S. Ultrasonic neuromodulation. J Neural Eng. 2016;13: 031003.

56. Beisteiner R, Matt E, Fan C, et al. Transcranial pulse stimulation with ultrasound in Alzheimer's disease: a new navigated focal brain therapy. Adv Sci (Weinh). 2019;7: 1902583.

57. Burger IA, Muller J, Donati OF, et al. ${ }^{68} \mathrm{Ga}$-PSMA-11 PET/MR detects local recurrence occult on mpMRI in prostate cancer patients after HIFU. J Nucl Med. 2019;60: 1118-1123.

58. Eiber M, Nekolla SG, Maurer T, Weirich G, Wester HJ, Schwaiger M. ${ }^{68}$ Ga-PSMA PET/MR with multimodality image analysis for primary prostate cancer. Abdom Imaging. 2015;40:1769-1771.

59. Melzer A, Gutmann B, Remmele T, et al. Innomotion for percutaneous image-guided interventions: principles and evaluation of this MR- and CT-compatible robotic system. IEEE Eng Med Biol Mag. 2008;27:66-73.

60. Berger J, Unger M, Landgraf L, Melzer A. Evaluation of an IEEE 11073 SDC connection of two KUKA robots towards the application of focused ultrasound in radiation therapy. Curr Dir Biomed Eng. 2019;5:149. 